\title{
Bristle Motion, Forces, and Related Vertical Translation for a Novel Electric Toothbrush Design
}

\author{
Frank Goldschmidtboeing ${ }^{1,}{ }^{*}-$ Uwe Pelz ${ }^{1}$-Karen Claire-Zimmet ${ }^{2}-$ Michael Wolf $^{2}$ - Ralf Goerlach ${ }^{2}-$ Peter Woias $^{1}$ \\ 1 University of Freiburg, Department of Microsystems Engineering, Germany \\ 2 Procter \& Gamble Service GmbH, Germany
}

This paper presents a combination of theoretical and experimental techniques applied to characterize the bristle motion, forces, and related vertical translation for a novel electric toothbrush design with a linear drive system. Results of the theoretical description, based on a single filament, were successfully compared with laboratory-based investigations: force measurements and high-speed video analysis, and tracking the toothbrush motion. This work describes the vertical translation induced in the toothbrush head, of up to $250 \mu \mathrm{m}$, when the toothbrush bristles are applied against a contact surface at brushing loads of approximately $1 \mathrm{~N}$ to $2.5 \mathrm{~N}$. Using these techniques, including Fast-Fourier transform analysis, it is shown that the vertical motion of the head is composed of the driving frequency and its harmonics.

Keywords: single filament theory, electric toothbrush, bristle motion, bristle forces, inclined toothbrush filaments

Highlights

- Single filament based theory for tooth brushing considiring bristle driven vertical translation of the brush head.

- Theoretical prediction and experimental validation of bristle peak forces at turning points of the oscillating-rotating motion.

- High-speed videography visualisation of brush head motion.

- Frequency analysis of the brush head motion for different drive types.

\section{O INTRODUCTION}

It may be surprising to learn that toothbrush designs have evolved over centuries. What may seem, at first thought, to be quite simple devices, are a key component of mechanical plaque removal and, therefore, oral hygiene in developed regions globally. Furthermore, far from being simple, many improvements have been made in how toothbrushes aid in plaque removal, including the introduction of electric toothbrushes in the mid-20th century.

In general, electric toothbrushes include a motor and power source in the handle, along with a gear system that drives the mechanical motion of the toothbrush head and the bristles. Electric toothbrushes vary in design, including not only the type of motion delivered, but also toothbrush head size and shape, and even in the size, type, and arrangement of bristles included within the head.

A wide variety of electric toothbrush models are currently found on the market, with by and large two main types of brush motion seen. One type of motion can be described as utilizing oscillating-rotating motion, in which the brush head (typically round or slightly oval) moves back and forth, at a given angle, around the central point of the brush head bristle surface. A second type of motion, often called "sonic" by toothbrush manufactures, typically involves a rectangular or oblong shaped head, which moves back and forth by rotating at a given angle relative to the long axis of the handle.

The cleaning efficacy and benefits to oral health by electric toothbrushes [1] to [5], and in particular rechargeable toothbrushes with oscillatingrotating motion [6] and [7], have been extensively demonstrated via clinical measurement techniques.

Additional developments in electric toothbrushes include features to aid user performance, including for instance toothbrush timers, which assist the user in brushing for an appropriate duration. More recent developments include pressure sensors which may respond, including signalling to the user, when brushing is performed at a given, higher pressure threshold - thereby reducing time spent brushing with excessive force [8]. Most recently, electric toothbrushes have also been developed that incorporate position detection and interactivity with smartphone apps, helping users brush not only with appropriate duration and pressure but also ensuring they brush all areas in the mouth evenly [9] and [10].

Regarding toothbrushes with oscillating-rotating $(\mathrm{O} / \mathrm{R})$ motion, the mechanical drive systems used to move the toothbrush head have also evolved. In the 1990s, Oral-B launched a novel drive system which introduced a driven three-dimensional (3D) pulsation in addition to $\mathrm{O} / \mathrm{R}$ motion, which has been demonstrated to improve cleaning efficacy via both laboratory [11] and clinical [12] testing methodologies. 
While cleaning efficacy is typically examined by clinical studies that are published in dentistry journals, there is almost no literature on the mechanical foundations or physics of toothbrush filament motion. This lack of publicly available, peer-reviewed research is likely due to the complicated interaction of the different filaments with each other and with the complex tooth surface. Our approach is to model single filaments and to determine their displacement and interaction force with the tooth. While the interaction between different filaments is neglected, high-speed videos show that toothbrush filaments typically move synchronously and, therefore, a basic model may provide insight, to aid in understanding the behaviour of the complex system.

This approach was also used in [13]. Regarding toothbrush head design, a study on the impact of bristle positioning in an $\mathrm{O} / \mathrm{R}$ head, and specifically the angling of bristles, has been presented with a semi-analytical model and discussion on impact to brushing shear force and penetration depth [13]. In that work, a filament with fixed length $L$ was treated as a cantilever, and the model assumed a fixed distance between the brush and a planar tooth surface $d$. (see Fig. 1).

Beyond the developments described above, further work has been performed to improve electric toothbrush designs, with a twofold goal: increased performance and improved brushing experience.

To accomplish this goal, a completely new motor and drive train was designed, incorporating a linear motor drive system, which enables a higher brush frequency and operates with a constant oscillation angle even under load.

In developing the new motor drive system, extensive technical, consumer, and clinical testing [14] to [17] were performed. Elements under investigation included overall brush motion, vibration and related experience perception, all of importance, particularly since the $3 \mathrm{D}$ pulsation drive introduced previously is not included within the new drive train. This work explains theoretical and experimental methods applied to describe and characterize the vertical translation, and related bristle motion and forces, for this novel electric toothbrush design.

\section{METHODS}

The main novelty in the theoretical description is the instruction of a bristle-driven brush head motion into the semi-analytic theory developed in [13]. In that publication, the distance between the brush head and tooth surface $d$ was assumed to be constant. However, high-speed videography shows that $d$ oscillates by typically about a few $100 \mu \mathrm{m}$. We have changed the boundary conditions of our model to account for this observation. The handle is still kept at a fixed position, but the brush head can be moved due to the finite stiffness of the handle. We furthermore do not label the different load cases by their distance $d$ but by their average force that is applied by the user to the tooth.

This average normal force $F_{N 0}$ compresses the filament such that the brush-head-to-tooth surface distance takes the value $d_{0}$. During the filament movement, the normal force $F_{N}$ and the distance $d$ might change. These changes can be related to each other by assuming a linear elastic behaviour of the handle.

$$
F_{N}=F_{N 0}+K \cdot\left(d-d_{0}\right)
$$

Here $K$ is the scaled spring constant of the handle, i.e., the ratio of the normal force of a single filament to the brush head displacement divided by the number of filaments. This approximation implies that all filaments of a particular type act synchronously on the single brush head.

This assumption is a simplification that renders the complex problem accessible for a single filamentbased theory.

As a typical brush head consists of filaments of different dimensions and inclinations, the different filaments act with different forces on the brush head, which is not covered in Eq. (1). Nevertheless, one type of filament typically dominates over the other types, which makes this simplification reasonable. Second, though inertial forces do not play an essential role for a single filament, the brush itself has a considerable mass, so that inertia plays a role for the brush head dynamics. This will lead to a delayed vertical translation of the brush head compared to this simplified theory.

Furthermore, the geometric relation between projected length $L_{x}$, tip displacement $w_{\text {tip }}$ and brushhead-to-tooth surface distance $d$ holds as in [8] (Fig. 1):

$$
d=\cos \alpha L_{x}-w_{t i p} \cdot \sin \alpha
$$




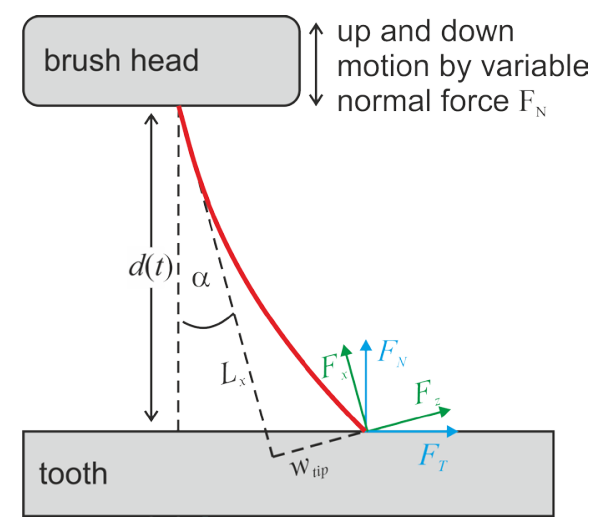

Fig. 1. Sketch of the theoretically examined filament shapes

We distinguish between the local coordinate system of the filament, where $x$ is the coordinate along the filament and $z$ is the coordinate perpendicular to it and the local coordinate system of the tooth surface with shear force $F_{T}$ and normal force $F_{N}$. Both coordinate systems are linked by the angle $\alpha$ (Fig. 1). The forces $F_{x}$ and $F_{z}$ are expressed by the shear force $F_{T}$ and the average normal force $F_{N 0}$ with the help of the Eqs. (1) and (2).

$$
\begin{aligned}
F_{x} & =F_{T} \cdot \sin \alpha+ \\
& +\left(F_{N 0}+K \cdot\left(\cos \alpha L_{x}-w_{t i p} \cdot \sin \alpha-d_{0}\right)\right) \cdot \cos \alpha,
\end{aligned}
$$

$$
\begin{aligned}
F_{z}= & F_{T} \cdot \cos \alpha+ \\
& +\left(F_{N 0}+K \cdot\left(\cos \alpha L_{x}-w_{\text {tip }} \cdot \sin \alpha-d_{0}\right)\right) \cdot \sin \alpha,
\end{aligned}
$$

Now the filament's bending line can be expressed in terms of $L_{x}, w_{\text {tip }}$ and $F_{T}$ by solving the EulerBernoulli-equation, including normal force.

$$
\begin{aligned}
& w\left(x, w_{\text {tip }}, L_{x}, F_{T}\right)=\frac{w_{\text {tip }}}{\tan \left(\lambda \cdot L_{x}\right)-\lambda \cdot L_{x}} . \\
& \cdot\left\{\sin (\lambda \cdot x)-\lambda \cdot x+\tan \left(\lambda \cdot L_{x}\right) \cdot(1-\cos (\lambda \cdot x))\right\}, \\
& \lambda^{2}=\frac{F_{T} \cdot \sin \alpha}{E I}+ \\
& \quad+\frac{\left(F_{N 0}+K \cdot\left(\cos \alpha L_{x}-w_{\text {tip }} \cdot \sin \alpha-d_{0}\right)\right) \cdot \cos \alpha}{E I} .
\end{aligned}
$$

To obtain a bending line that only depends on one parameter, two equations have to be found to express the mutual dependence of $L_{x}, w_{\text {tip }}$ and $F_{T}$. With these equations, all parameters (i.e., forces and the bending line) of the filament can be expressed in dependence on tip displacement $w_{\text {tip }}$.

The first equation states that the bending force at the tip $F_{z}$ calculated from the bending line Eq. (5) equals that from Eq. (3). The second equation is given by the fact that the filament length does not change. Here $E I$ is the flexural rigidity of the bristle.

$$
\begin{gathered}
E I \cdot w_{t i p} \cdot \frac{\lambda^{3}}{\lambda \cdot L_{x}-\tan \left(\lambda \cdot L_{x}\right)}=F_{T} \cdot \cos \alpha- \\
-\left(F_{N 0}+K \cdot\left(\cos \alpha L_{x}-w_{t i p} \cdot \sin \alpha-d_{0}\right)\right) \cdot \sin \alpha, \\
L-\int_{0}^{L_{x}} \sqrt{1+\frac{d w^{2}}{d x}} d x \approx L-L_{x}+\frac{1}{2} \frac{d w^{2}}{d x}=0 .
\end{gathered}
$$

As mentioned, solving Eqs. (7) and (8) for given $w_{\text {tip }}$ yields all information about the filament's forces and displacements.

The above calculations are all done under the assumption that the average distance $d_{0}$ is known. We assume that the average distance $d_{0}$ is the same as the static distance at the same normal force. In static conditions, the tangential force $F_{T}$ should be near zero, which means that the static case is given by a displacement $w_{\text {tip }}$ that fulfils Eq. (9).

$$
F_{T}=0 .
$$

From this solution, the equilibrium brush-headto-tooth surface distance $d_{0}$ and the average normal force per bristle $F_{N 0}$ can be calculated.

When modelling the motion of the filaments, it turns out that the tangential stiction force $F_{\text {stic }}$ has to be considered, as it delivers the reason that the filament's tip position becomes stuck at each turning point of the rotational motion. The filament can only move along the tooth surface as long as it overcomes the maximum stiction force $F_{\text {stic }}$. A common model for the stiction force is given by Eq. (10).

$$
\left|F_{\text {stic, } \max }\right|=\mu \cdot F_{N} \text {. }
$$

Here $\mu$ is the static friction coefficient. The stiction force is negative, if the filament moves in a forward direction, i.e., from left to right in Fig. 1, and consequently positive for backward movement. Solving Eq. (10) with $F_{\text {stic, } \max }=F_{T}$ results in the maximum normal force per bristle $F_{N \text {, max }}$. If a bristle moves from a turning point in a forward direction, the tip becomes stuck until the resulting shear force $F_{T}$ overcomes the maximum stiction force in a negative direction, according to Eq. (10). Consequently, the positive maximum stiction force has to be overcome if coming from a turning point and moving in a backward direction. When the maximum stiction force is overcome, dynamic friction occurs. Its value 
depends not only on the normal force but also on the velocity.

Furthermore, a typical brush head consists of tufts with different inclinations, directions, and even different filament lengths. The full quantitative modelling of this complicated friction pattern is extremely challenging; nevertheless, a simplified model that describes the main features of the brush head is useful to understand and optimize the brushing performance.

As an example, we model the new toothbrush head (Fig. 2) used with the linear drive system, which has approximately $n_{F}=2900$ bristles angled in the forward direction, $n_{B}=600$ bristles angled in the backward direction and $n_{0}=200$ non-inclined bristles. To further simplify the model, we assume a constant length of $L=8.8 \mathrm{~mm}$, a radius of $r=76 \mu \mathrm{m}$ and an inclination angle of $\alpha=15^{\circ}$ for each filament, though a few filaments are slightly longer. The filaments are made of polyamide (PA 6.12), which has a Youngs' modulus of $E=2.7 \mathrm{GPa}$. A friction coefficient of $\mu=0.3$ was assumed.

Most of the time, all the bristles will exert a total normal force $F_{N, \text { min }}$ on the tooth. At the respective turning points, forces of $F_{N 1}$ and $F_{N 2}$ will occur. Therefore, we expect two force peaks with the height of $F_{N 1}$ and $F_{N 2}$ with a phase shift of $180^{\circ}$.

$$
\begin{gathered}
F_{N, \text { min }}=\left(n_{F}+n_{B}+n_{0}\right) \cdot F_{N 0}, \\
F_{N, 1}=\left(n_{B}+n_{0}\right) \cdot F_{N 0}+n_{F} \cdot F_{N, \max }, \\
F_{N, 2}=\left(n_{F}+n_{0}\right) \cdot F_{N 0}+n_{B} \cdot F_{N, \text { max }} .
\end{gathered}
$$

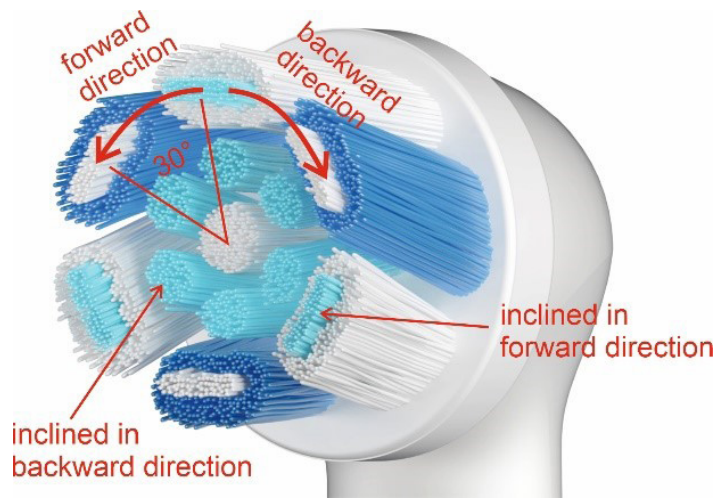

Fig. 2. Filament pattern of the novel brush head

\section{EXPERIMENTAL}

The experimental methods to examine the bristle driven head motion and the related forces are twofold: First, the motion of the brush head was visualized and tracked by high-speed video imaging; second, force measurements were conducted.

To achieve repeatable conditions, all measurements were done under dry conditions, i.e., without water or toothpaste. Toothpaste and water will have an impact on the friction coefficient and the Youngs' modulus of the filaments. Therefore, we expect that the forces while cleaning will be lower than those measured in our laboratory set-up.

In preparation for high-speed videography, laserstructured marking dots (ø $450 \mu \mathrm{m})$ and scale bars $(1930 \mu \mathrm{m})$ on tape were carefully attached to relevant moving and stationary parts of the brush heads, enabling scaled movement tracking within the video frames (Fig. 3).
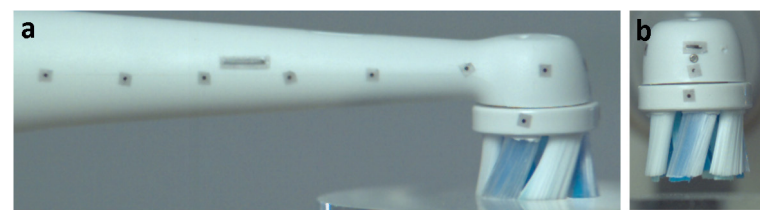

Fig. 3. Brush head with tracking markers in a) side- and b) front view

The brush handle was secured in two pairs of custom, 3D printed clamps, ensuring the possibility for vibration only in the brush head. The clamps were attached to an aluminium stand, which was mounted to a movable stage. By using the stage movement capabilities and pressing the brush head on a flat, polished acrylic block on top of a scale (Sartorius, ED822), the force applied to the brush head could be adjusted (Fig. 4).

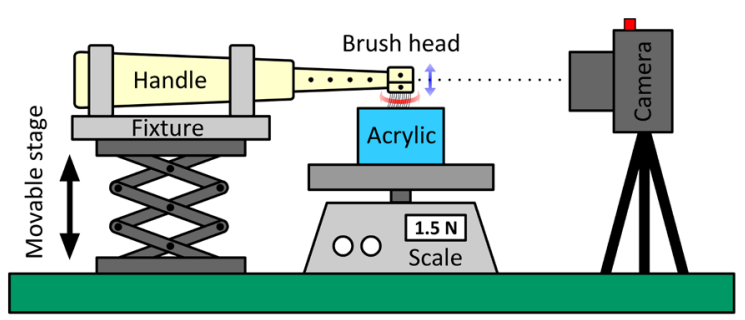

Fig. 4. Measurement set-up for high-speed videography 


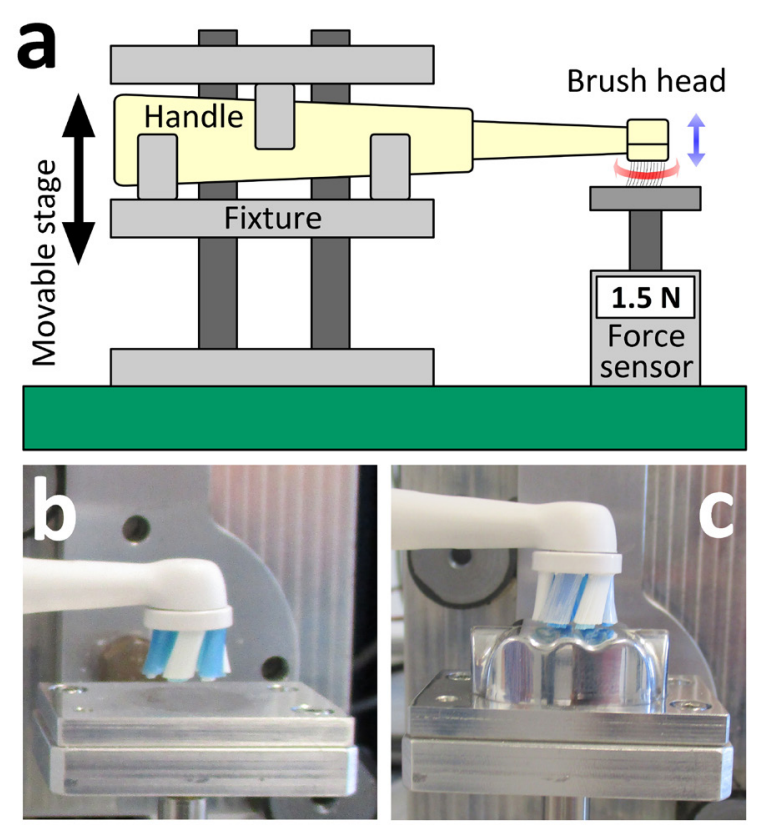

Fig. 5. a) Measurement set-up for transient force measurements; b) using a flat or c) profiled surface

High-speed video of the brush head in front and side view was recorded on a Chronos 1.4 highspeed camera from Kron Technologies Inc. (Burnaby, Canada) at 4000 frames per second.

All recordings have been analysed using the freeto-use software Tracker (www.physlets.org/tracker/, version 5.0.6) to extract the relative movement of all micro markers in each frame.

A similar set-up was used for the force measurement (Fig. 5). The brush is pressed to a flat aluminium plate (Fig. 5b) and alternatively on an aluminium plate with a tooth-like topography (Fig. $5 \mathrm{c})$. The aluminium plate is fixed on a force sensor (Kistler Model 9217A) with response threshold of $1 \mathrm{mN}$. The senor signal is amplified with a Kistler Model 5018 charge amplifier and recorded with a sample time of $50 \mu \mathrm{s}$. The brush head is free to move according to its elasticity as only the other end of the handle is fixed. All experiments were recorded for a total duration of $2 \mathrm{~s}$. The averaged normal force $F_{N 0}$ is determined by averaging the transient force signal.

\section{RESULTS \& DISCUSSION}

The vertical translation of the brush head and the rotation angle $\phi$ versus time for the average normal force between $1 \mathrm{~N}$ and $2.5 \mathrm{~N}$ are shown in Fig. 6. The periodicity of the up-and-down motion (black curve) is the same as that of the linear drive (red curve), but the force peaks are delayed compared to the driving signal. As mentioned in the theory part, this is attributed to the inertia of the brush head.

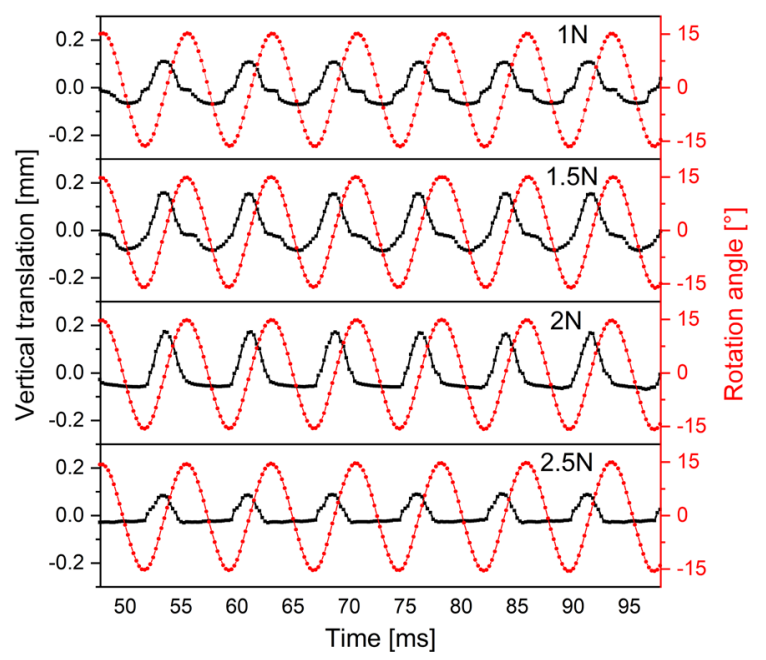

Fig. 6. Vertical translation (black) and rotation angle (red) versus time

Furthermore, the total stroke versus average normal force is plotted in Fig. 7. The difference between the maximum and minimum vertical translation was determined for each period and averaged for the whole signal. Theory predicts an increase of stroke with increasing normal force. The experiment, however, shows a maximum value of about $250 \mu \mathrm{m}$ and a decreasing stroke for forces higher than $2 \mathrm{~N}$. This is explained by a sidewise bulging of the filaments, which is not modelled by the theory.

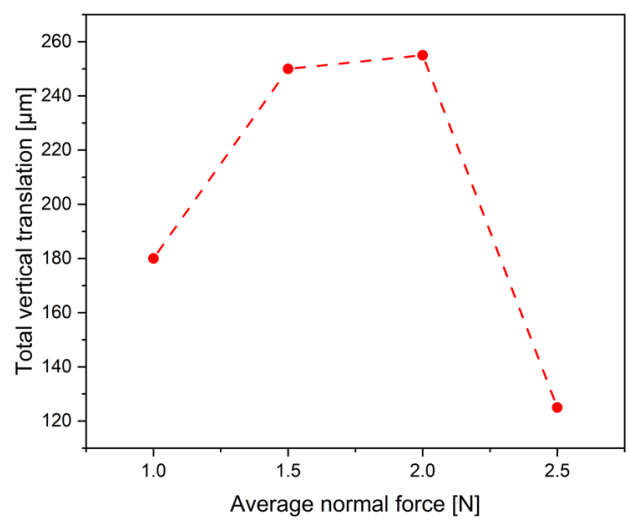

Fig. 7. Total vertical stroke vs. average normal force $F_{N 0}$

A more direct comparison of the experiment to theory can be made from the force data.

Fig. 8 shows a time series of force data for an average normal force of $2 \mathrm{~N}$. The black curve shows 
the unfiltered force data and the red marks show the automatically detected force peaks with a circular shape for the force $F_{N 1}$, and a diamond shape for the force $F_{N 2}$. The dashed lines show the averaged values.

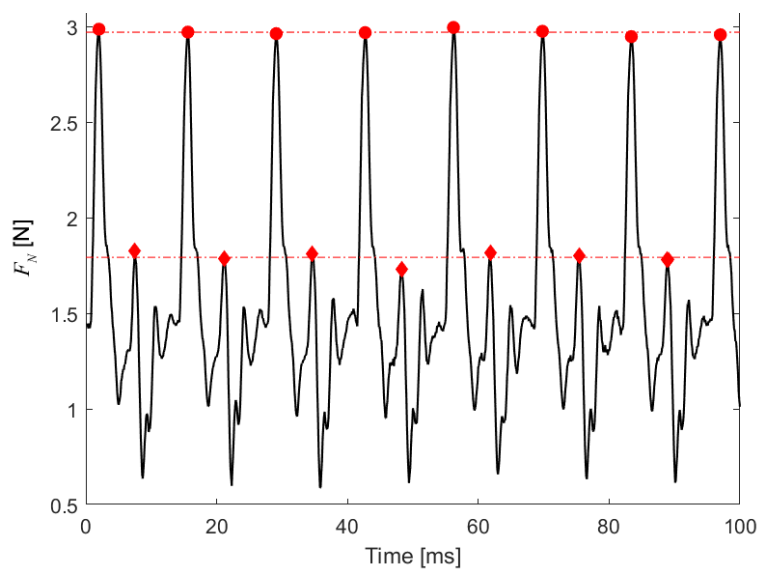

Fig. 8. Transient force data for the novel brush head at an average normal force of $2 \mathrm{~N}$

These data show much more details compared to the displacement data, as short spikes of force do not directly transform to displacement due to the inertia of the brush head.

This transient curve shows two force maxima per period which are separated by exactly half a period and show two different values. These values are interpreted as force maxima $F_{N 1}$ (Eq. (12)) for the turning point to forward motion and $F_{N 2}$ (Eq. (13)) for the turning point to backward motion. The averaged experimental values for these force peaks and the theoretical predictions are compared in Fig. 9, where the red marks indicate the force maximum $F_{N 1}$ while the black marks indicate the force maximum $F_{N 2}$. Each point was measured three times to assess the repeatability. The lines indicated the theoretical values (Eqs. (12) and (13)). The results scatter significantly, as it was relatively difficult to adjust the same conditions in every experiment. Nevertheless, both curves match well, especially for high forces. The experimental maximum forces, however, show relatively high deviations at the lowest normal force of $1 \mathrm{~N}$. The reason for this deviation is probably a vibration of the sensor plate itself that was observed at low normal force values.

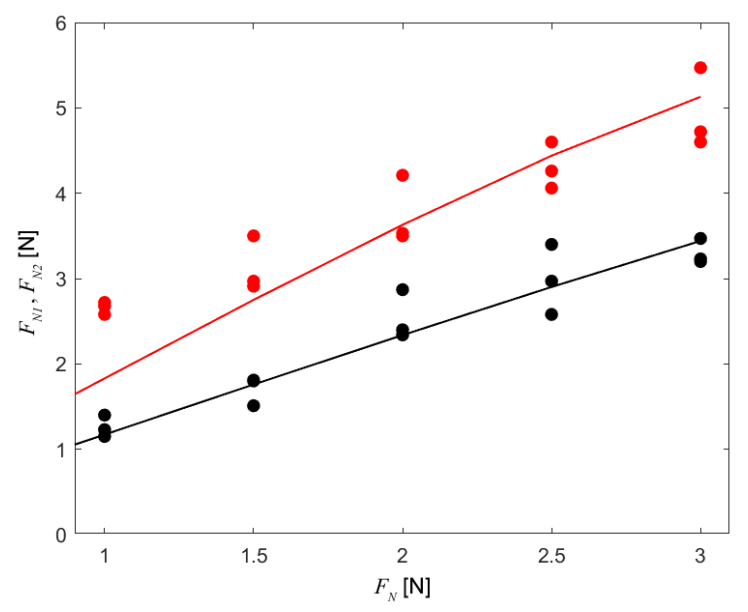

Fig. 9. Comparison of experimental and theoretical magnitudes of the force peaks at the turning points

To further examine the vibrations of the brush head under realistic conditions, force measurements were conducted on the aluminium plate with the toothlike topography. Fig. 10 shows a fast Fourier transform (FFT) of the force data for an average force of $2 \mathrm{~N}$ with a linear $\mathrm{O} / \mathrm{R}$ dive. Only the drive frequency of about $147 \mathrm{~Hz}$ and its harmonics are present.

Fig. 11 shows the FFT of a comparable experiment with a brush with additional 3D pulsation (see [8] for details on the brush design). In this case, both drives, the $\mathrm{O} / \mathrm{R}$ drive with a frequency of $93 \mathrm{~Hz}$ and the $3 \mathrm{D}$ pulsation drive with a frequency of $417 \mathrm{~Hz}$ interact nonlinearly, which leads to complicated frequency pattern with many different frequency components in the sensible frequency range of a few hundred Hz. Not only those two mismatched frequencies but also the sums and difference of those and their multiples are present.

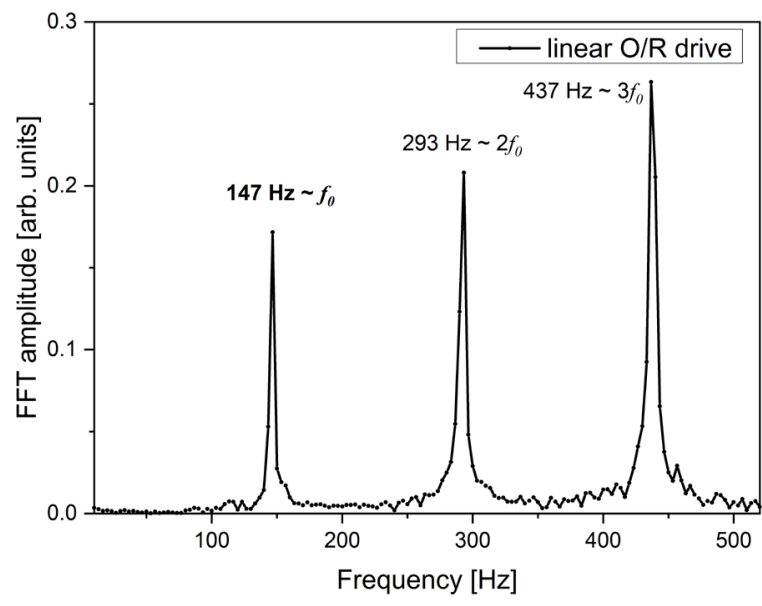

Fig. 10. FFT analysis of the frequency spectrum of the normal force with the novel brush design and the linear drive 


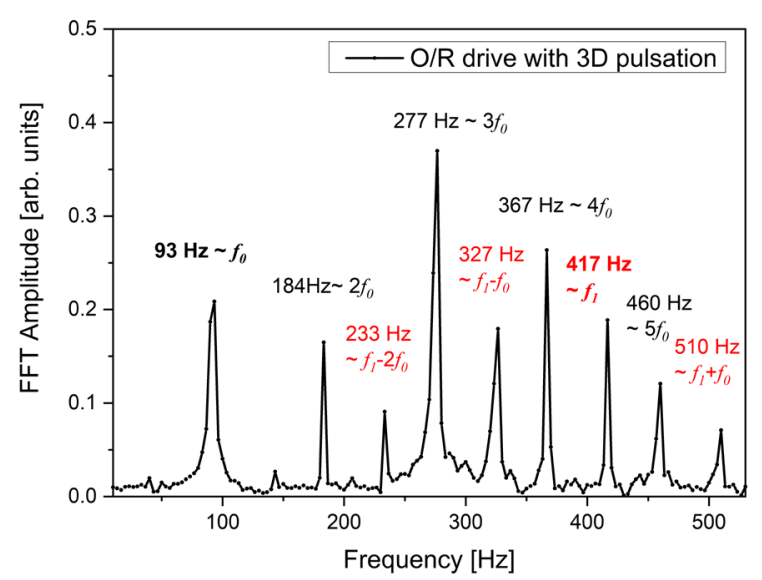

Fig. 11. FFT analysis of the frequency spectrum of the normal force with the $0 / R$ drive with $3 D$ pulsation

\section{CONCLUSIONS}

A single filament model was utilized to describe the complex bristle motion of an electric toothbrush, including the effect of bristle driven vertical translation of the brush head. The main predictions of the simple model, such as the value of the force peaks at the turning points, the periodicity and the order of magnitude of the vertical translation were confirmed by the experiment. Thus, the simple model helps the designers of toothbrushes to optimize their design. Other details, such as the reduction of vertical translation amplitude at high average normal forces or the exact transient behaviour of the brush head were experimentally examined while the model was not capable of predicting these effects quantitively, because effects like sidewise bulging and the inertia of the brush were not included into the single-filamentbased model. Therefore, a mixed theoretical and experimental approach is necessary to design and understand the complex movement patterns of driven brush heads.

The experiments have shown that an electric brush with linear $\mathrm{O} / \mathrm{R}$-drive produces force peaks at the turning points that can be adjusted by the ratio of filaments inclined into different directions. A vertical translation of up to $250 \mu \mathrm{m}$ amplitude is generated by the novel design. This amplitude is influenced by properties, such as the stiffness, orientation, and quantity of filaments, as well as the handle stiffness, and can therefore be modulated. The current value has been perceived as pleasing by test consumers. The force signal of the brush with linear drive consists only of the drive frequency and its harmonics.

Though toothbrushes are in daily use and have a history of centuries, there are still open questions that may be addressed by theoretical and experimental methods.

\section{ACKNOWLEDGEMENTS}

This work was funded by Procter \& Gamble Service GmbH, Germany.

\section{REFERENCES}

[1] Rosema, N., Slot, D.E., van Palenstein Helderman, W.H., Wiggelinkhuizen, L., Van der Weijden, G.A. (2016). The efficacy of powered toothbrushes follong a brushing exercise: a systematic review. International Journal of Dental Hygiene, vol 14, no. 1, p. 29-41, D0l:10.1111/idh.12115.

[2] Heanue, M., Deacon, S.A., Deery, C., Robinson, P.G., Walmsley, A.D., Worthington, H.V., Shaw, W.C., Shaw, B.C. (2003). Manual versus powered toothbrushing for oral health. Cochrane Database of Systematic Reviews, no. 1, art. no. CD002281, DOI:10.1002/14651858.cd002281.

[3] Yaacob, M., Worthington, H.V., Deacon, S.A., Deery, C., Walmsley, A.D., Robinson, P.G., Glenny, A.M. (2014). Powered versus manual toothbrush for oral health. Cochrane Database of Systematic Reviews, no. 6, art. no. CD002281, DOI:10.1002/14651858.CD002281.pub3.

[4] Van der Weijden, F.A., Slot, D.E. (2015). Efficacy of homecare regimens for mechanical plaque removal in managing gingivitis a meta review. Journal of Clinical Periodontology, vol. 42, no. S16, p. S77-S91, D0l:10.1111/jcpe.12359.

[5] Pitchika, V., Pink, C., Völzke, H., Welk, A., Kocher, T., Holtfreter, B. (2019). Long-term impact of powered toothbrush on oral health: 11-year cohort study. Journal of Clinical Periodontology, vol. 46, no. 7, p. 713-722, D0l:10.1111/jcpe.13126.

[6] Grender, J., Adam, R., Zou, Y. (2020). The effects of oscillatingrotating electric toothbrushes on plaque and gingival health: A meta-analysis. American Journal of Dentistry, vol 33, no. 1, p. 3-11.

[7] Clark-Perry, D, Levin, L. (2020) Systemic review and metaanalysis of randomized controlled studies comparing oscillating rotating and and other powered toothbrushes. The Journal of the American Dental Association, vol. 151, no. 4, p. 265-275, D0I:10.1016/j.adaj.2019.12.012.

[8] Janusz, K., Nelson, B., Bartizek, R.D., Walters, P.A., Biesbrock, A. (2008). Impact of a novel power toothbrush with SmartGuide technology on brushing pressure and thoroughness. Journal of Contemporary Dental Practice, vol. 9, no. 7, p. 1-8.

[9] Erbe, C., Klees, V., Ferrari-Peron, P., Cahuana-Vasquez, R.A., Timm, H., Grender, J., Cunningham, P., Adam, R., Farrell, S., Wehrbein, H. (2018). A comparative assessment of plaque removal and toothbrushing compliance between a manual and an interactive power toothbrush among adolescents: a single-center, single-blind randomized controlled trial. BMC Oral Health, vol. 18, no. 1, p. 130, D0l:10.1186/s12903-0180588-1.

[10] Erbe, C., Klees, V., Braunbeck, F., Ferrari-Peron, P., CcahuanaVasquez, R.A., Timm, H., Grender, J., Cunningham, P., Adam, R., Wehrbein, H. (2019). Comparative assessment of plaque 
removal and motivation between a manual toothbrush and an interactive power toothbrush in adolescents with fixed orthodontic appliances: A single-center, examiner-blind randomized controlled trial. American Journal of Orthodontics and Dentofacial Orthopedics, vol. 155, no. 4, p. 462-472, DOI:10.1016/j.ajodo.2018.12.013.

[11] Driesen, G.M., Warren, P.R., Hilfinger, P. (1998). Cleaning efficacy of a new electric toothbrush. American Journal of Dentistry, vol. 11, special issue, p. S7-S11.

[12] Ernst, C.P., Nauth, C., Willershausen, B., Warren, P.R. (1998). Clinical plaque removing efficacy of a new power toothbrush. American Journal of Dentistry, vol. 11, special issue, $p$. S13-S16.

[13] Goldschmidtboeing, F., Doll, A., Neiss, S., Doll A., Stoerkel, U., Woias, P. (2014). Comparison of vertical and inclined toothbrush filaments: Impact on shear force and penetration depth. Strojniški vestnik - Journal of Mechanical Engineering, vol. 60, no. 7-8, p. 449-461, D0l:10.5545/sv-jme.2013.1513.

[14] Adam, R. (2020). Introducing the Oral-B i0 electric toothbrush: next generation oscillating-rotating technology. International
Dental Journal, vol. 70 , no. S1, p. S1-S6, D0l:10.1111/ idj.12569.

[15] Grender, J., Goyal, C.R., Qaqish, J., Adam, R. (2020). An 8-week randomized controlled trial comparing the effect of a novel oscillating-rotating toothbrush versus a manual toothbrush on plaque and gingivitis. International Dental Journal, vol. 70, No. S1, p. S7-S15, D0l:10.1111/idj.12571.

[16] Adam, R., Goyal, C.R., Qaqish, J., Grender, J. (2020). Evaluation of an oscillating-rotating toothbrush with microvibrations versus a sonic toothbrush for the reduction of plaque and gingivitis: results from a randomized controlled trial. International Dental Journal, vol. 70, no. S1, p. S16-S21, D0l:10.1111/idj.12569.

[17] Adam, R., Erbe, J., Grender, J. (2020). Randomized controlled trial assessing plaque removal efficacy of a novel oscillatingrotating electric toothbrush with micro-vibrations. International Dental Journal, vol. 70, no S1, p. S22-S27, D0l:10.1111/ idj.12568. 